\title{
Bilateral subclavian steal syndrome in an intensive care unit. A case report.
}

\author{
Hrvoje Budincevic ${ }^{1}$, Latica Friedrich¹, Nikolina Tolj-Karaula², Nikolina Maric², Tena Sucic ${ }^{3}$, \\ Ivan Bielen ${ }^{1}$
}

${ }^{1}$ Stroke and Intensive Care Unit, Department of Neurology, ${ }^{2}$ Department of Internal Medicine, ${ }^{3}$ Department of Radiology, University Hospital „Sveti Duh“, Zagreb, Croatia

\begin{abstract}
We report the case of a 56-year-old man who presented with arterial hypotension, lightheadedness, vomiting, a sense of tingling in his right arm, and a right-beating horizontal nystagmus. He was initially admitted to the Intensive care unit and treated with standard vasopressor agents. A neurosonological examination showed the steal phenomenon on both vertebral arteries; the neuroradiological examination revealed occlusion of the left subclavian artery and subtotal stenosis of the innominate artery. Percutaneous transluminal angioplasty was performed. Our case demonstrates how bilateral subclavian steal syndrome should be taken into account in the case of a seemingly hypotensive patient unresponsive to standard therapy.
\end{abstract}

Keywords: subclavian steal syndrome, arterial hypotension, ultrasound

\section{Introduction}

Subclavian steal syndrome is a group of symptoms caused by a proximal stenosis or the occlusion of a subclavian or innominate artery [1]. This leads to a reversal of blood flow in the vertebral artery in order to provide blood supply to the ipsilateral arm [2]. The symptoms are due to the "steal" of blood supply to the brain and include vertebrobasilar symptoms such as paroxysmal vertigo, dizziness, diplopia, ataxia, dysarthria and syncopal attacks [2]. Symptoms can also be the consequence of ischemia to the hand, i.e. arm weakness, claudication, paresthesias or coldness in the arm $[3,4]$. However, frequently patients

Received 13.04.2014 Accepted 15.05.2014

Med Ultrason

2014, Vol. 16, No 3, 264-267

Corresponding author: Hrvoje Budinčević, M.D.

Stroke and Intensive Care Unit.

Department of Neurology, University Hospital

„Sveti Duh“, Zagreb, Croatia.

64, Sveti Duh street

HR-10000, Zagreb, Croatia

Phone: +385 13712 159, Fax: +385 13712372

E-mail: hbudincevic@gmail.com with this condition have no neurologic symptoms, up to $95 \%[5,6]$. The condition can therefore be suspected only based on a marked blood pressure difference between the two arms [2,7]. Prevalence of subclavian steal syndrome is reported between 0.6 and $6.4 \%$ in the ultrasonographical studies [5-8], and among those a bilateral condition seems to be rare, around 4\% [7]. This case report reveals a potential cause of falsely low values of arterial blood pressure unresponsive to standard vasopressor treatment.

\section{Case report}

A 56-year-old man presented to the Emergency department with sudden lightheadedness, vomiting and a sense of tingling in his right arm. He reported feeling worse after self-administration of $5 \mathrm{mg}$ isosorbide dinitrate sublingually. His medical history included a myocardial infarction 9 years prior to this event and a lumbar spine surgery due to a herniated disc 11 years ago. He had also been taking antihypertensive medications until 3 years ago when his blood pressure allegedly normalized and currently was not taking any medications regularly. He smoked approximately 30 cigarettes per day. 
At presentation his arterial blood pressure (BP) was $80 / 60 \mathrm{mmHg}$ on both arms. The neurologic examination was normal except for a right-beating horizontal gaze-evoked nystagmus and a sense of tingling in his right arm. The brain computed tomography (CT) scan and chest X-ray were normal. Electrocardiogram (ECG) showed an incomplete right bundle branch block. He had a raised blood glucose level of $9.1 \mathrm{mmol} / \mathrm{L}$, elevated leukocytes $\left(13.06 \times 10^{\wedge} 9 / \mathrm{L}\right), \mathrm{CRP}(7.7 \mathrm{mg} / \mathrm{L})$, cholesterol $(6.85 \mathrm{mmol} / \mathrm{L})$, LDL-cholesterol $(5.23 \mathrm{mmol} / \mathrm{L})$, and triglycerides $(2.9 \mathrm{mmol} / \mathrm{L})$ while the rest of the laboratory tests which included RBC count, platelets, electrolytes, AST, ALT, CK, CK-MB, troponine, urea and creatinine were normal.

He was admitted to the Intensive care unit with the working diagnosis of iatrogenic arterial hypotension and vasopressor therapy with dopamine was started. The BP was continously invasively monitored (with the canule in the left brachial artery) as well as the central venous pressure (with the catether in the right jugular vein), and vasopressor and volume replacement intravenous therapy was titrated according to the current values. However, the arterial BP remained unresponsive to the therapy. Clinically, an audible bruit over left carotid artery, as well as in left supra- and infraclavicular region was noted. The patient's BP was $160 / 80 \mathrm{mmHg}$ on both legs at the popliteal arteries. Differential diagnosis included a possible aortic dissection or a myocardial infarction, partially supported by an elevation of plasma CK-MB from 13 to 52 $\mathrm{U} / \mathrm{L}$ after a 5 hour interval; however, his troponin levels remained $0 \mu \mathrm{g} / \mathrm{L}$ with a normal heart ultrasound and no dynamics in the ECG. CT angiography of the aorta was interpreted as normal, and the vasopressor treatment was stopped. A carotid and vertebral duplex scan was then performed which showed signs of partial steal phenomenon in the right common carotid artery (CCA), with reversed flow in the right external carotid artery (ECA) and reduced flow in the right internal carotid artery (ICA). There were signs of bilateral steal phenomenon in the vertebral arteries (fig 1-3).

Transcranial Doppler (TCD) pointed to reversed flow in both vertebral arteries in their intracranial segment. CT angiography was repeated, this time with the imaging of the supra-aortic arteries, and showed atherosclerotic plaques at the proximal segment of innominate artery, a narrow right CCA $(3.5 \mathrm{~mm})$ and a possible left subclavian artery obliteration at the proximal segment. Both vertebral arteries were poorly visualized (fig 4).

A vascular surgeon and interventional radiologist were summoned, and together with the neurologist decided in favor of an endovascular treatment. While waiting for the procedure, the patient was discharged from the hospital and prescribed acetylsalicylic acid $100 \mathrm{mg}$ daily and atorvastatin $20 \mathrm{mg}$ due to hyperlipidaemia.

Eventually, a percutaneous transluminal angioplasty on innominate artery was performed. The digital subtraction angiography (DSA) of the aortic arch and supra-aortic arteries was performed prior to the endovascular treatment and confirmed a subtotal stenosis of the innominate artery and occlusion of the left subclavian artery. The left subclavian artery was visualized via branches of left ECA, and right subclavian artery via distal part of right CCA. By selective left CCA catheterization and contrast infusion a complete circle of Willis and intracranial arteries were visualized which showed no signs of pathomorphological changes. Clopidogrel $75 \mathrm{mg}$, perindopril $2 \mathrm{mg}$ and bisoprolole $1.25 \mathrm{mg}$ daily were added to the prescribed therapy. Two months after the procedure a control carotid and vertebral arteries ultrasound showed only ultrasound signs of a complete left subclavian steal syndrome. Neurological examination of the patient was normal and he had no symptoms except for the occasional tingling sensations in his left arm.

\section{Discussion}

Subclavian steal syndrome is often an incidental duplex ultrasonography finding in an asymptomatic patient $[5,9]$. Its presence can be suspected based on a brachial arterial pressure difference [2], but in a clinical setting blood pressure is rarely measured on both arms [10]. A symptomatic bilateral subclavian syndrome is an even less frequent condition [7], so it is not commonly suspected. In our patient with known cardiovascular risks (male gender, hypertension, hyperlipidemia and smoking), the bilateral reduced flow in the vertebral arteries was severe enough to provoke the signs and symptoms of the posterior cerebral circulation (nystagmus, vomiting and vertigo). However, the falsely low BP measured over both brachial arteries and the application of the hypotensive agents prior to the medical presentation led to the belief that hypotension was the cause of our patient's symptoms. A further delay in the correct diagnosis was the result of the initial CT aortography which was interpreted as normal, because only the aortal arch was analyzed, without a review of the supra-aortic vessels. An ultrasound of the carotid and vertebral arteries was crucial for establishing the final diagnosis.

When analyzing the cause of our patient's syndrome, one possibility was Takayasu arteritis [2,11]. The American College of Rheumatology 1990 criteria for the diagnosis of Takayasu arteritis requires 1) angiographic criteria to show narrowing or occlusion of the entire aorta, its primary branches, or large arteries in the proximal upper 


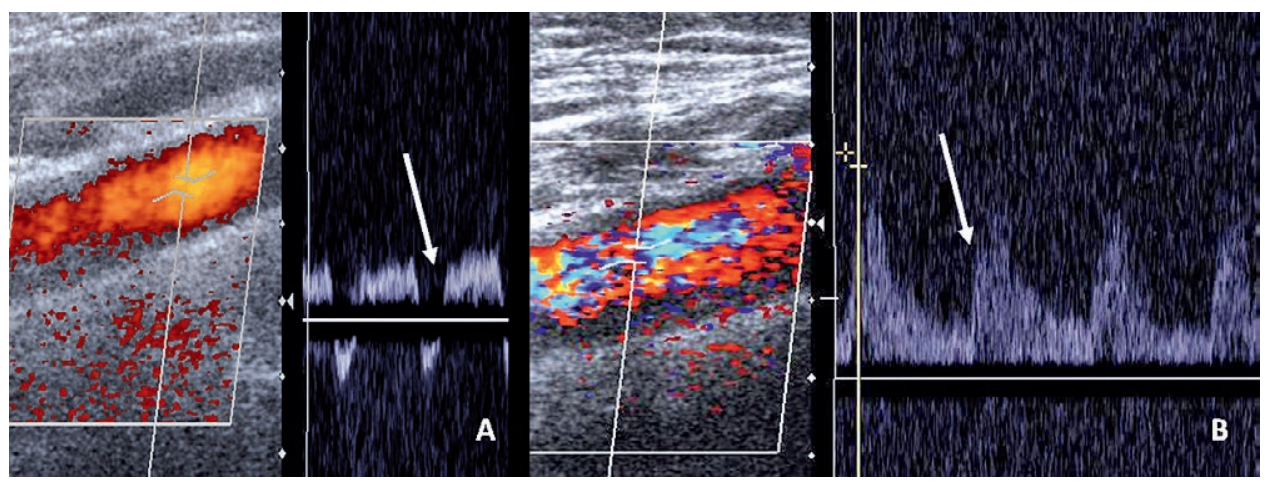

Fig 1. Duplex ultrasound of right common carotid artery: a) before percutaneous transluminal angioplasty showing steal phenomonenon - retrograde flow during systole (arrow): b) after percutaneous transluminal angioplasty showing normal waveforms (arrow).

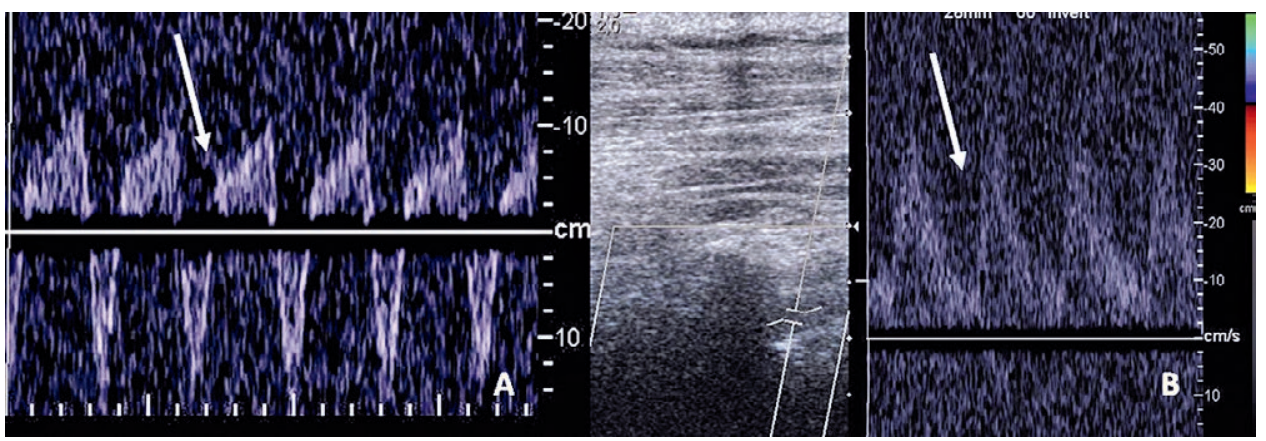

Fig 2. Duplex ultrasound of right vertebral artery: a) before percutaneous transluminal angioplasty showing partial steal phenomonenon - retrograde flow through right vertebral artery during systole (arrow); b) after percutaneous transluminal angioplasty showing normal waveforms (arrow).

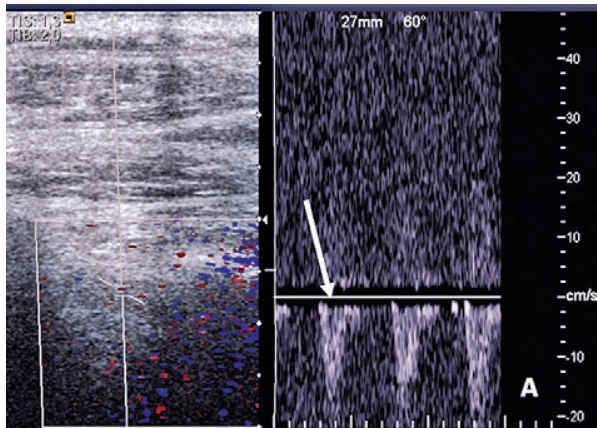

Fig 3. Duplex ultrasound of left vertebral artery showing complete subclavian steal phenomenon - retrograde flow through left vertebral artery (arrow).

or lower extremities, 2) that the changes are usually focal or segmental and 3) that these changes are not due to arteriosclerosis, fibromuscular dysplasia, or similar causes [12]. While the first two criteria were met, it is more probable that the stenosis of the arteries in this case were due to arteriosclerosis [2] . This is supported by our

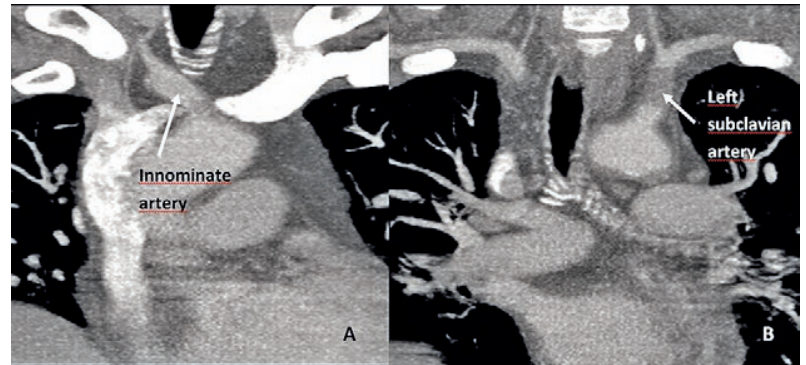

Fig 4. CT angiography (CTA) of aortic arch: a) atherosclerotic plaques with stenosis at the proximal segment of innominate artery (arrow); b) occlusion of the left subclavian artery.

patients age, previous myocardial infarction, hypertension, hyperlipidaemia, smoking, and also by the presence of atherosclerotic plaques on the ultrasound. Takayasu arteritis is also more common in females and presents for the first time in patients over 40 years only in $10-20 \%$ of cases [13]. 
This case report highlights the importance of including bilateral subclavian steal syndrome to the differential diagnostic procedure of a hypotensive patient with neurological symptoms. These patients should have their BP measured over both arms and even legs when medical history and clinical presentation raise the suspicion of this condition. Ultrasonography of the arteries of the neck is a simple, fast and convenient method of screening for this syndrome in a symptomatic patient [2]. Once the diagnosis is made, the syndrome can be successfully treated, as was the case with our patient.

\section{References}

1. Psillas G, Kekes G, Constantinidis J, Triaridis S, Vital V. Subclavian steal syndrome: neurotological manifestations. Acta Otorhinolaryngol Ital 2007; 27: 33-37.

2. Osiro S, Zurada A, Gielecki J, Shoja MM, Tubbs RS, Loukas M. A review of subclavian steal syndrome with clinical correlation. Med Sci Monit 2012; 18: RA57-RA63.

3. Taylor CL, Selman WR, Ratcheson RA. Steal affecting the central nervous system. Neurosurgery 2002; 50: 679-688; discussion 688-679.

4. Smith JM, Koury HI, Hafner CD, Welling RE. Subclavian steal syndrome. A review of 59 consecutive cases. J Cardiovasc Surg (Torino) 1994; 35: 11-14.
5. Labropoulos N, Nandivada P, Bekelis K. Prevalence and impact of the subclavian steal syndrome. Ann Surg 2010; 252: 166-170.

6. Hennerici M, Klemm C, Rautenberg W. The subclavian steal phenomenon: a common vascular disorder with rare neurologic deficits. Neurology 1988; 38: 669-673.

7. Tan TY, Schminke U, Lien LM, Tegeler CH. Subclavian steal syndrome: can the blood pressure difference between arms predict the severity of steal? J Neuroimaging 2002; 12: 131-135.

8. Fields WS, Lemak NA; Joint Study of extracranial arterial occlusion. VII. Subclavian steal--a review of 168 cases. JAMA 1972; 222: 1139-1143.

9. Bornstein NM, Norris JW. Subclavian steal: a harmless haemodynamic phenomenon? Lancet 1986; 2: 303305.

10. Asai Y, Kawamoto R, Nago N, Kajii E. Blood pressure measurement by primary care physicians: comparison with the standard method. Nihon Koshu Eisei Zasshi 2000; 47: 326-336.

11. Numano F, Kobayashi Y. Takayasu arteritis--beyond pulselessness. Intern Med 1999; 38: 226-232.

12. Arend WP, Michel BA, Bloch DA, et al. The American College of Rheumatology 1990 criteria for the classification of Takayasu arteritis. Arthritis Rheum 1990; 33: 11291134.

13. Kerr GS, Hallahan CW, Giordano J, et al. Takayasu arteritis. Ann Intern Med 1994; 120: 919-929. 